\title{
Communicating earthquake risk: mapped parameters and cartographic representation
}

\author{
J. M. Gaspar-Escribano and T. Iturrioz \\ ETSI Topografía, Geodesia y Cartografía, Universidad Politécnica de Madrid, Spain
}

Received: 25 June 2010 - Revised: 27 October 2010 - Accepted: 11 November 2010 - Published: 8 February 2011

\begin{abstract}
Earthquake risk assessment is probably the most effective tool for reducing adverse earthquake effects and for developing pre- and post-event planning actions. The related risk information (data and results) is of interest for persons with different backgrounds and interests, including scientists, emergency planners, decision makers and other stakeholders. Hence, it is important to ensure that this information is properly transferred to all persons involved in seismic risk, considering the nature of the information and the particular circumstances of the source and of the receiver of the information. Some experience-based recommendations about the parameters and the graphical representations that can be used to portray earthquake risk information to different types of audiences are presented in this work.
\end{abstract}

\section{Introduction}

The assessment of natural risks for emergency response and preparedness planning is a horizontal discipline in the sense that it can be studied from many perspectives, including those of social, political and Earth sciences. Natural risks affect every individual to some extent, including people with different profiles and backgrounds (Fig. 1). Hence, the use of a common language is indispensable to improve the understanding of the processes related to potential adverse situations. In the end, this would mean a better collective preparedness and response capability to natural disaster (e.g., Bostenaru Dan, 2005; Haque and Etkin, 2007). Firstly, this requires the identification of the obstacles for an efficient communication between the parties (Reckelhoff-Dangel and Petersen, 2007). A correct dissemination of information depends on several factors related to the actual awareness about

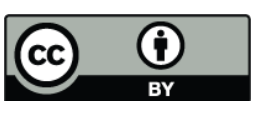

Correspondence to:

J. M. Gaspar-Escribano

(jorge.gaspar@upm.es) a given natural risk (if I am not aware of something, how can I be prepared to give a proper response to it?), the degree of understanding about it (if I do not get the information in a way that I understand it, how can I learn positive lessons from it?) and the interest for getting the information (is it more convenient to tackle the problem with full consequences or just to ignore it and assume the aftermath?).

Secondly, once the communication obstacles have been identified, measures to overcome them may be formulated. Two aspects are considered: the parameters used to represent the information that is reckoned relevant and the graphical representation of the information to be conveyed. The graphical representation provides a powerful instrument for understanding the information, as well as making it attractive and, hence, to reach a broader audience. Geographic Information Systems (GIS), whose use on natural risk studies is very extended, constitute ready tools for this purpose (e.g., Anagnostopoulosa et al., 2008).

In this work, the issues introduced above are examined using earthquake risk as an example of natural risk. In contrast to volcanic eruptions, which are limited to a roughly reduced extension, or to meteorological phenomena (heavy storms, tornados, floods) which can be forecast with enough time for taking information/protection/evacuation measurements, nowadays earthquake occurrence is impossible to predict accurately in time and space (e.g., Geller et al., 1997). Only probabilistic estimates of earthquake occurrence, which involve very high uncertainties, may be put forward (Gerstenberger et al., 2005). Although these uncertainties may be reduced in some cases (densely instrumented, eagerly investigated areas, such as populated cities located in regions of high seismic activity; areas affected by a noticeable earthquake during the immediate hours/days succeeding its occurrence) they are far from actual earthquake occurrence predictions. The practical impossibility of predicting earthquakes makes earthquake risk assessment one of the most useful tools for preventing undesired

Published by Copernicus Publications on behalf of the European Geosciences Union. 


\author{
NATURE OF INFORMATION \\ AND SEISMIC CONTEXT \\ Regional scale seismic risk analysis \\ in low seismic activity areas
}

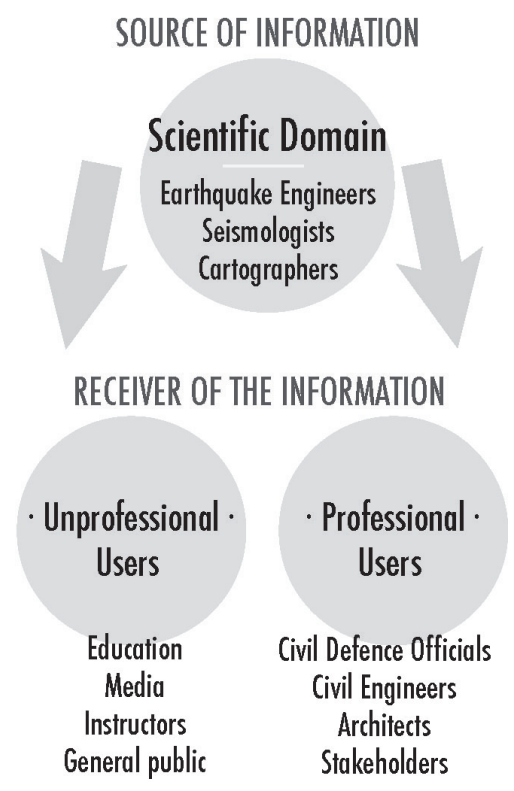

Fig. 1. Chart showing the scope of this work and the flow of information.

earthquake effects and planning pre- and post-event actions (Coburn and Spence, 2002).

Earthquake risk is the aggregate of several facets: seismic hazard (expected seismic ground motion), structure vulnerability (susceptibility to withstand or not earthquake shaking), exposure of persons and structures, related damage and costs. They all must be taken into account during the analysis and the communication of seismic risk analyses and results. Four factors influencing earthquake risk communication are commented below in this paper: the seismic environment, the nature of information, the source of the information produced and transferred and the receiver of the information (Fig. 1).

An important issue on natural risk communication is related to uncertainty assessment. Uncertainty is inherent to natural phenomena and, therefore, it should be incorporated into the communication chain as part of the message, especially when it takes significant values.

\section{Earthquake risk features and factors influencing communication}

The actual seismic environment of the study area is the first factor conditioning the correct dissemination of earthquake risk-related information. Whereas in areas of high seismic activity such as California, initiatives such as the Great California Shakeout (a massive drill and coordinated actions to get the population and organizations prepared against earthquake effects, http://www.shakeout.org/) are put forward, in low activity areas such events are not as frequent and extensive just to keep the population aware about the actual risk situation.

The nature of the information refers to the scope and characteristics of the seismic risk analysis, based of the information. Depending on the scale and degree of detail, earthquake risk studies can be classified in regional (small scale) and local/urban (large scale). Regional seismic risk analyses are used for information and educational purposes as well as establishing initial (preliminary) risk assessments that form the basis for further detailed studies, such as ranking of areas with higher/lower risk for the definition of future actuation priorities. By contrast, the general purpose of urban seismic risk analyses are related to the quantification of repair/retrofit, reconstruction and reinforcement costs (preevent), response planning (post-event) and urban planning (future developments). By definition, regional risk studies are less detailed than local risk studies. Whereas qualitative estimates may be valid for regional risk studies, quantitative estimates are essential for urban risk analyses. As the regional approach is naturally accomplished ahead of the local approach, efforts to minimize problems of communication and of information dissemination should be emphasized, especially in regional risk assessment approaches.

The source of the information may be a scientific article, a mass media publication, a confidential report for stakeholders, etc. Differences on information sources are related to different approaches to manipulate and elaborate the information, depending on the interest of the author and of its client.

The last, but not least, factor influencing communication is the receiver. Evidently, it is not the same to report to a professional user of the information than to an unprofessional user. Professional users, such as urban planners, architects, civil engineers, risk insurers, Civil Protection managers and other stakeholders, have the responsibility of using and understanding this information correctly, otherwise the results of their work could lead to adverse consequences to the society. The remainder of individuals or social groups, such as mass media, instructors and general public, is included in the category of unprofessional users. An unprofessional user typically has limited information on the subject of natural risks.

\section{Concepts of graphic semiology and thematic cartography}

Communication between the various agents involved in the knowledge of the seismic characteristics of a region (from scientists to the wide end-user spectrum) is mainly carried 

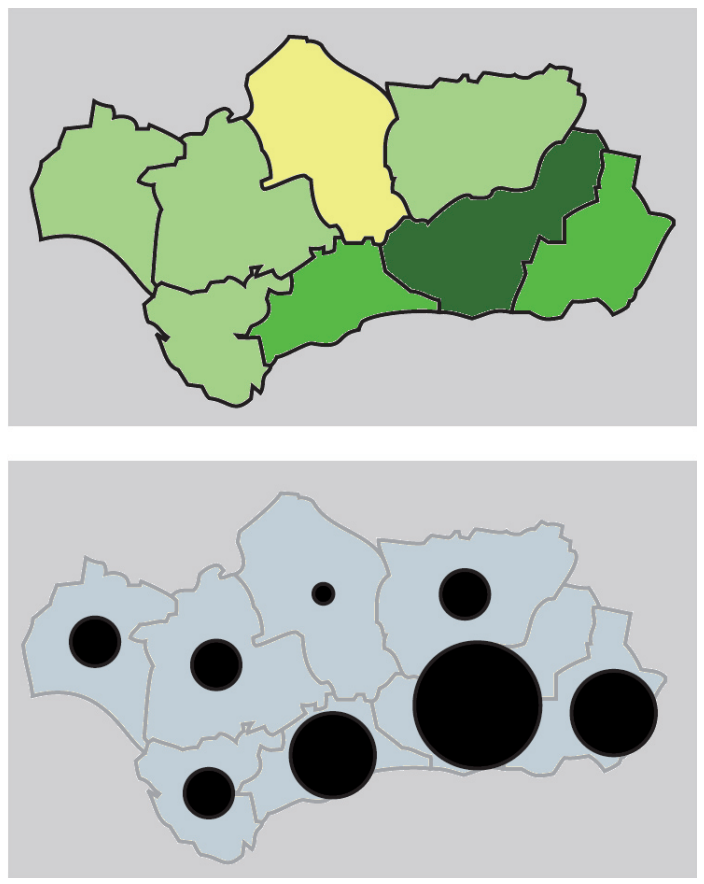

Fig. 2. Maps representing the same quantitative variable with colours (top) and symbols (bottom). Although no explanatory legend is included, darker colours and bigger symbols are perceived as meaning higher values.

out through maps. The particularities of typical parameters and their spatial distribution are explained and seized by means of a specific cartography; the correct reception of the seismological message will depend on the design of this cartography.

The improvement of this understanding must, therefore, tackle cartography from the perspective of visual communication and pay special attention to map design. This perspective of cartography as a communication vehicle is well known by cartographers, being an important subject for cartographic literature (Dent, 1972; Robinson and Petchenik, 1977; MacEachren, 1995; Slocum et al., 2005) for decades, since Bertin first set up the template for graphic semiology (Bertin, 1967).

Not all graphic signals are perceived in the same way, but our perception reacts differently depending on which type of graphic stimulus is considered. Darker colours and bigger symbols are naturally considered as meaning higher (stonger, larger, etc) values than lighter colours and smaller symbols (Fig. 2, Bertin, 1976). These two kinds of visual hierarchies are perceived spontaneously, regardless of culture or nationality. What is more, these responses are really powerful, as it is difficult to go against them: it is hard for us to interpret the big as non-significant and the small as important when reading a map.
This hierarchical perception of size and grey-value makes it appropriate to employ these visual variables (size and value) in order to reflect quantitative and ordered data in maps. In seismological cartography, mostly quantitative data are depicted (qualitative data are directly derived from quantitative estimates), such as seismic hazard or expected damage maps. These general concepts of graphic semiology are the basis for thematic cartography and provide the grounds for the seismic risk mapping recommendations that are presented in this work.

\section{Objective and scope}

The scope of the study is restricted to regional-scale risk analyses in areas of low-to-moderate seismic activity, which is probably the scenario (seismic environment and nature of information) that demands more efforts to improve communication. Only the most basic risk situation, related to direct ground-shaking and physical damage of normal-importance buildings, is considered in the present analysis. This excludes concatenated earthquake effects; vulnerability and damage of infrastructures and lifelines; and social vulnerability related to lack of preparedness and resilience of the society as a whole.

In this work, the source of information is basically scientific and embraces contributions from seismologists, earthquake engineers and cartographers. Their roles are different and complement each other: Seismologists and earthquake engineers should define the parameters that are the most adequate to reach different target audiences and cartographers should define the characteristics of the final graphical representations that are easier to understand. Whereas a lot of discussion exists on how to bridge the communication gap between professionals with distinct backgrounds such as seismologists and engineers, little can be found on how cartographers may play a significant role in the improvement of communication of seismic risk assessment results to external users.

Finally, the receiver of the information considered in this work is varied. Professional users basically include urban planners and administration managers interested in (1) defining geographical areas (e.g., municipalities, counties) where developing more detailed, local-scale seismic risk analyses; (2) establishing priorities for assigning resources to different zones for preventative measurements. Unprofessional users are the rest of the individuals eventually interested in the topic: mass media, teachers or institutions with the goal of informing and educating the exposed population, among others.

In the remainder of the paper, we present some experiencebased guidelines or recommended practices aimed at facilitating communication on earthquake risk, which could be extrapolated to similar risk assessment studies of other natural phenomena. 


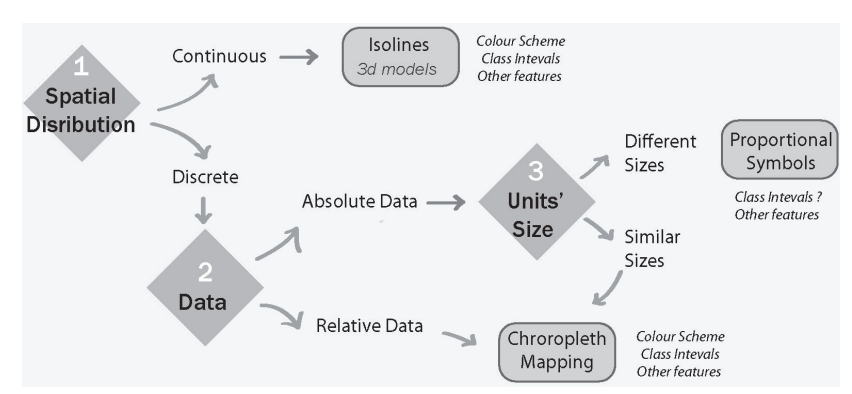

Fig. 3. Guidelines for graphical representation of geographical variables.

\section{Communicating earthquake risk: parameters}

The choice of parameters representing seismic hazard, vulnerability, expected damage and cost should be in agreement with the knowledge and expectations of the final receiver of the information. Whereas an unprofessional user would just be interested in knowing whether these risk factors take a high or a low value in a simple manner, a professional user would also be concerned at how they compare with the values contained in the seismic code or building normative and what is the cost/benefit ratio of implementing any risk-informed action. In this regard, an uncertainty estimate assessment should accompany the end-result.

Seismic hazard is usually computed on rock conditions (strongly controlled by seismicity) and then modified to include site effects (related to local topography and geology) yielding the seismic hazard including site effects. Most seismic codes include a seismic hazard map expressed in terms of expected peak ground acceleration (PGA) on rock conditions for a given exceedance probability level (some codes use other parameters such as spectral accelerations, SA). As these are the parameters used to regulate earthquake-resistant design of structures, it is important to make these parameters available to professional users, so they reckon how the outcome of the regional risk study compares with the values expressed in the normative. Additionally, for some seismic risk applications based on empirical relations among damage-vulnerability and ground motion, macroseismic intensity may be used (and has been extensively used in the past) as hazard parameter. However, these hazard parameters have no meaningful content for unprofessional audiences (actually, they may be misleading about the actual hazard). Then, for unprofessional audiences it may be preferable to provide a seismic hazard map in terms of other parameters informing about both the significant lateral variability of hazard estimates, although the actual value presented may contain higher uncertainties. Two of such parameters are the expected PGA including site effects and the intensity (related to observed damage rather than to ground motion). As probabilistic hazard estimates are difficult to internalize by
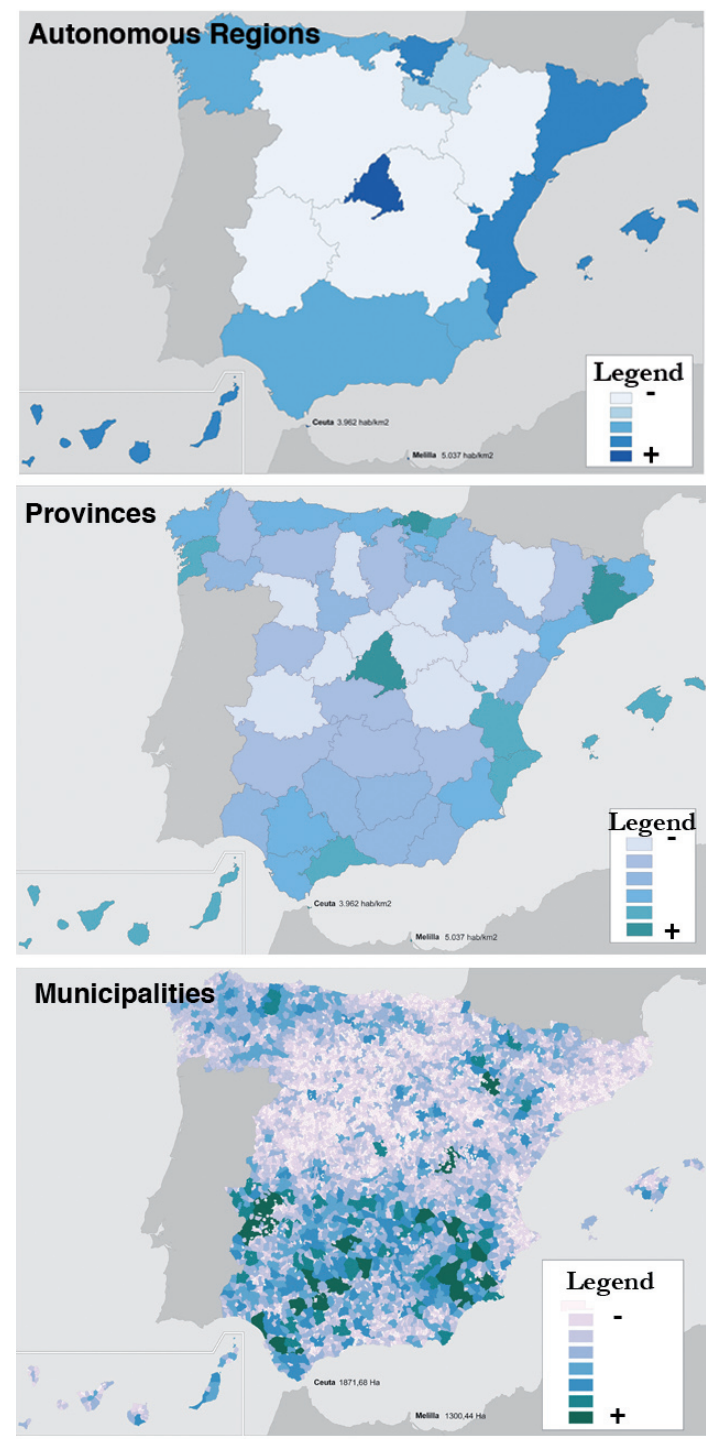

Fig. 4. The administrative units of Spain are, in decreasing size, Autonomous Regions (top), Provinces (middle) and Municipalities (bottom). The choice of a geographical representation unit presents a significant repercussion on the final aspect of the map.

unprofessional users (Mileti et al., 2004), it may be convenient to reorganize hazard results in a simple scale (such as high/medium/low hazard).

Features determining the communication of vulnerability and of damage results are very similar and, hence, can be treated jointly. A complete characterisation of the regional distribution of seismic vulnerability (and of expected damage) requires assessing absolute (total numbers) and relative estimates (percentages). For professional users, knowing both absolute and relative quantities is essential. Absolute amounts give a direct idea of the resources that are needed to devise and relative amounts inform about the overall vulnerability or the severity of the damage in its geographical 
context. For unprofessional users, the communication of vulnerability and of damage results should be less complex than for professional users. With this purpose, results should be reorganized according to new, straightforward classifications containing a few vulnerability classes and damage grades. Examples of such recommended classifications for predominant building vulnerability or expected damage degree of a municipality are high/medium/low average vulnerability or severe/moderate/slight damage degree.

The inclusion of cost estimates on earthquake risk assessment studies is interesting and important because it sheds light onto the economical impact of the catastrophic event and allows developing cost/benefit studies and informed preparedness planning. For professional users, both relative and absolute amounts of cost parameters may be needed. For instance, a civil engineer may need the assessing of the total cost of repair/reinforcement/retrofit to a given structure, whereas a civil protection planner may be interested in knowing the expected cost in relation to the annual budget available. By contrast, an unprofessional user of the cost information would better understand a cost estimate in relation to an average salary or in terms of extra taxes than a total cost that it is difficult to substantiate.

\section{Communicating earthquake risk: graphical representations}

Regardless of the actual meaning of the parameter, the first consideration that should be made for its graphical representation relates to whether the parameter follows a continuous or a discrete spatial distribution (Fig. 3). The reason is that continuous phenomena need to be mapped showing that continuity, for which - in bidimensional representations - isolines are required. Seismic hazard on rock conditions is the only continuous variable used in seismic risk analysis.

Discrete spatial distributions need different representation methods. Depending on whether data are absolute or relative amounts, the cartographical logic will lead to two main different representation forms: Proportional Symbols and Choropleth mapping (Robinson et al., 1995; Dent, 1999). The first technique - also known as Graduated Circles - is based on one single symbol, usually a circle, whose size changes according to the represented value. This technique admits the representation of both types of data - absolute and relative. Choropleth mapping uses a predefined colour scheme, shading the considered geographical units according to the value taken by the variable within the unit. In cartographic design, the use of Choropleth mapping is only appropriate for the representation of relative amounts (Fig. 2).

Given that both choropleth and proportional symbol mapping are used to depict discrete phenomena, they are based on geographical units or polygons for their representations (note that the representation geographical unit may differ from the actual unit where risk calculations were performed). The size
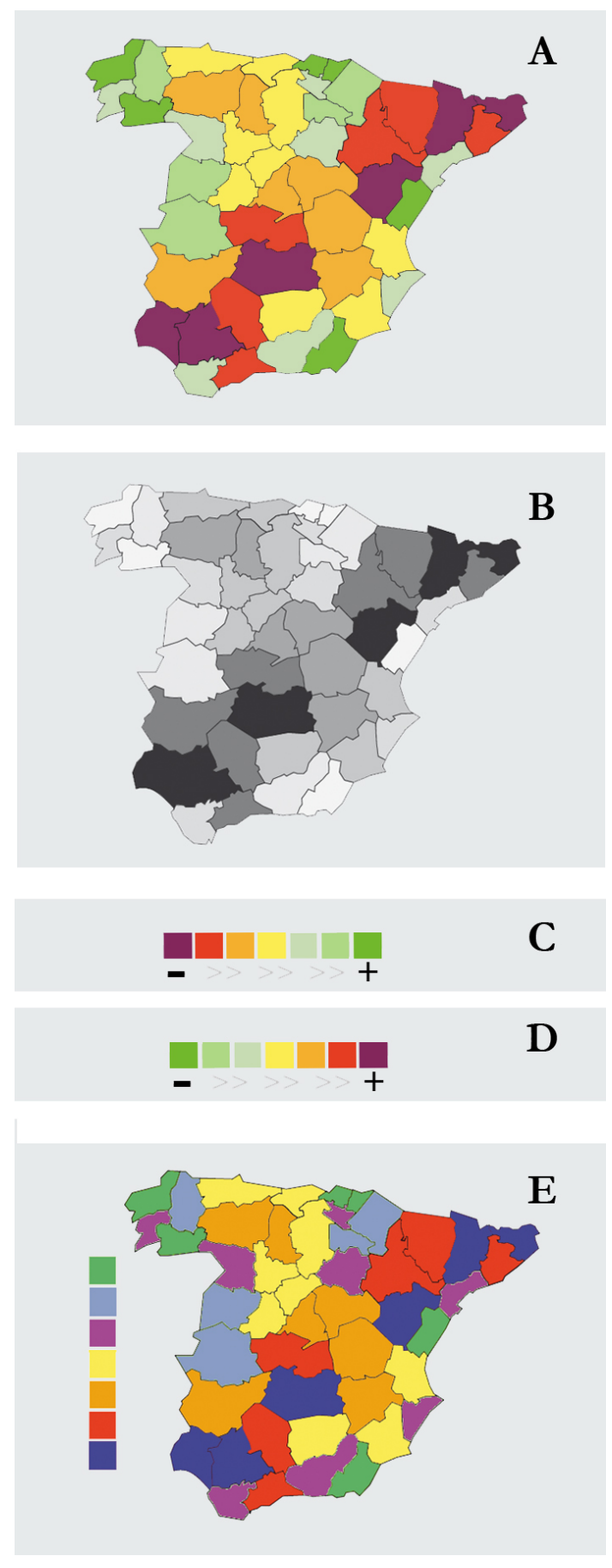

Fig. 5. Quantitative colour schemes for sequential quantitative variables. Maps (A) and (E) show the same information with a different colour scheme. With this colour scheme it is unclear which variable takes higher and lower values, unless a legend ( $\mathbf{C}$ or $\mathbf{D}$ for map $\mathbf{A})$ is included. Map (B) is a grey-scale version of map (A).

of these geographical units becomes crucial for the visualisation, as it determines the spatial variability shown in the map. Figure 4 shows three representations of the same variable as 

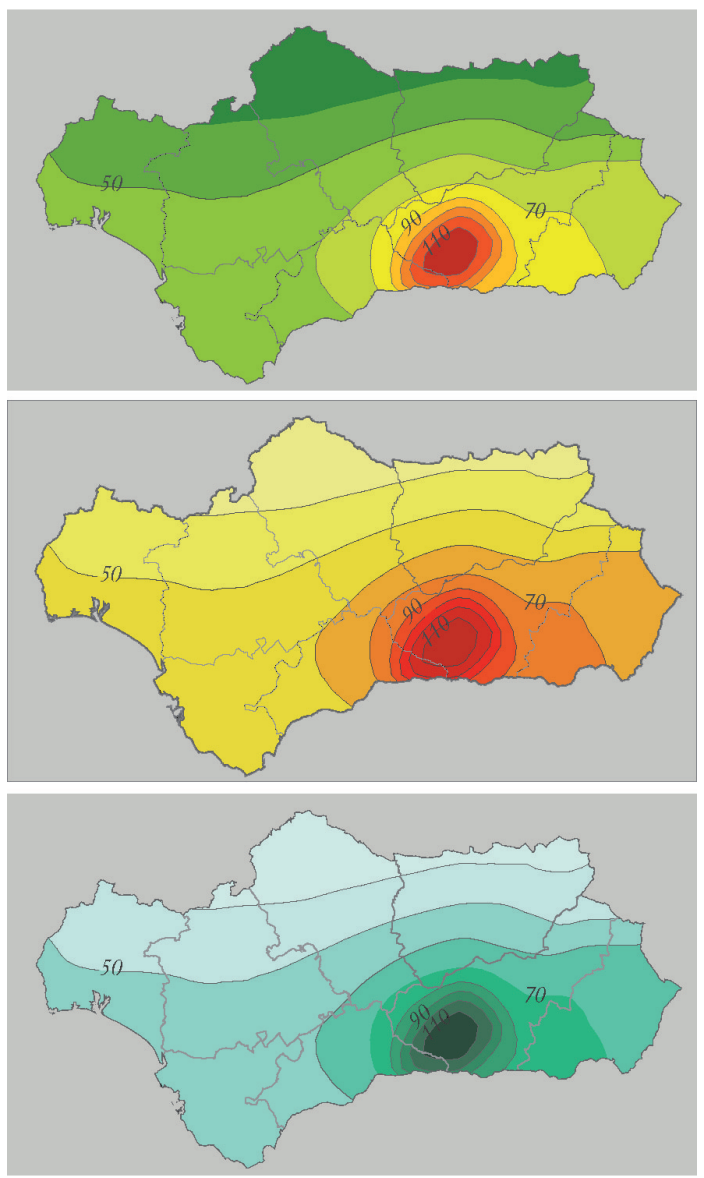

Fig. 6. Seismic hazard map on rock conditions using different colour patterns: top: traffic-light criterion; middle: two-colour grading; bottom: single-colour grading. The latter two options are recommended instead of the traffic-light criterion.

grouped in different geographical units. Whereas they all are valid, their visual aspect is very diverse. Excess and defect of detail, as expressed in the bottom and top panels, respectively, should be avoided if possible.

In our experience, the use of colour instead of size is preferable for the understanding of the seismological message (Top Fig. 2 preferred to bottom Fig. 2). Choropleths and isolines use colour as a vehicle for the transmission of quantitative information. The colour scheme selection must follow the natural human perception, where variations in colour grey-value or lightness, light and dark shades of just one selected hue (e.g., shades of blue, as in Fig. 4) are perceived as hierarchically ordered. For instance, take maps a and b in Fig. 5, which portray the same information and have no legend. Map b, based on grey scale only, reflects better the hierarchical order. However, this hierarchy is not easily extracted from Map a unless a legend is included (Fig. 5, panel c or d). Map e in Fig. 5 is even more difficult to interpret even if a legend is included.
Another example that is worth mentioning concerns the traffic-light criterion, which is widely used within the scientific community (Fig. 6, top). It typically adopts a threecolour scheme (red-yellow-green) in order to represent highmedium-low values (or moderate-strong-weak, or unsafeintermediate-safe). These colours differ in hue but coincide in grey-value. Therefore, they are not perceived with a hierarchical structure. This model is (over) confident on the power of the conventional, learnt, symbolic connotations of these colours, and neglects the hierarchical perception of the chiaroscuro effect. The final result may be confusing to the end-user (unless the three-colour scheme is combined with progressive darkness) and, thus, the plain use of the trafficlight criterion is not recommended (Brewer, 1997).

Apart from colour selection, another main point that is often overlooked when representing seismic hazard estimates (or, in general, continuous variable plots) is related to class intervals (Fig. 7). They must be chosen carefully, as they can dramatically change the image of the data provided by the map and, therefore, its interpretation (Evans, 1977; Cauvin et al., 2010). Although regular class limits are desirable, as they are easily understood, they usually do not adapt to the depiction of the original data distribution (Fig. 7). A statistical approach to data classification, such as the one provided by Jenks (Jenks and Coulson, 1963), currently implemented in cartographic software, conveys the actual variability of the represented parameter. Because of its consequent class irregularities, this advantage is normally achieved at the cost of the map reading (Fig. 7). Therefore, extreme care should be taken when using this classification method, as well as being aware of the nature of the end-user of the map. Eventually, seismic hazard can be reclassified in categories (in agreement with the significance of the parameter) in order to facilitate the interpretation of hazard maps in terms of high-hazard and low-hazard areas.

In any case, all decisions concerning map design must be made taking into account a very specific potential end-user. In this sense, a map in its classic conception will be limited to meeting the needs of one particular profile. In order to cover a broader spectrum of users, interactive cartographic representations can be proposed, as they allow users to tailor the map to their needs and cognitive profiles, selecting aspects such as the representation method, colour scale, interval classification, geographical unit definition and contextual information (Cartwright, 1997; Miller, 2007; Peterson, 2007). What is more, map animations and three-dimensional models can be integrated, thus, enriching the capabilities of cartography as a vehicle for communicating the seismic message to broad audiences.

\section{Discussion and conclusions}

Many times the research work is so absorbing and specialised that we miss other points that are very important to our 


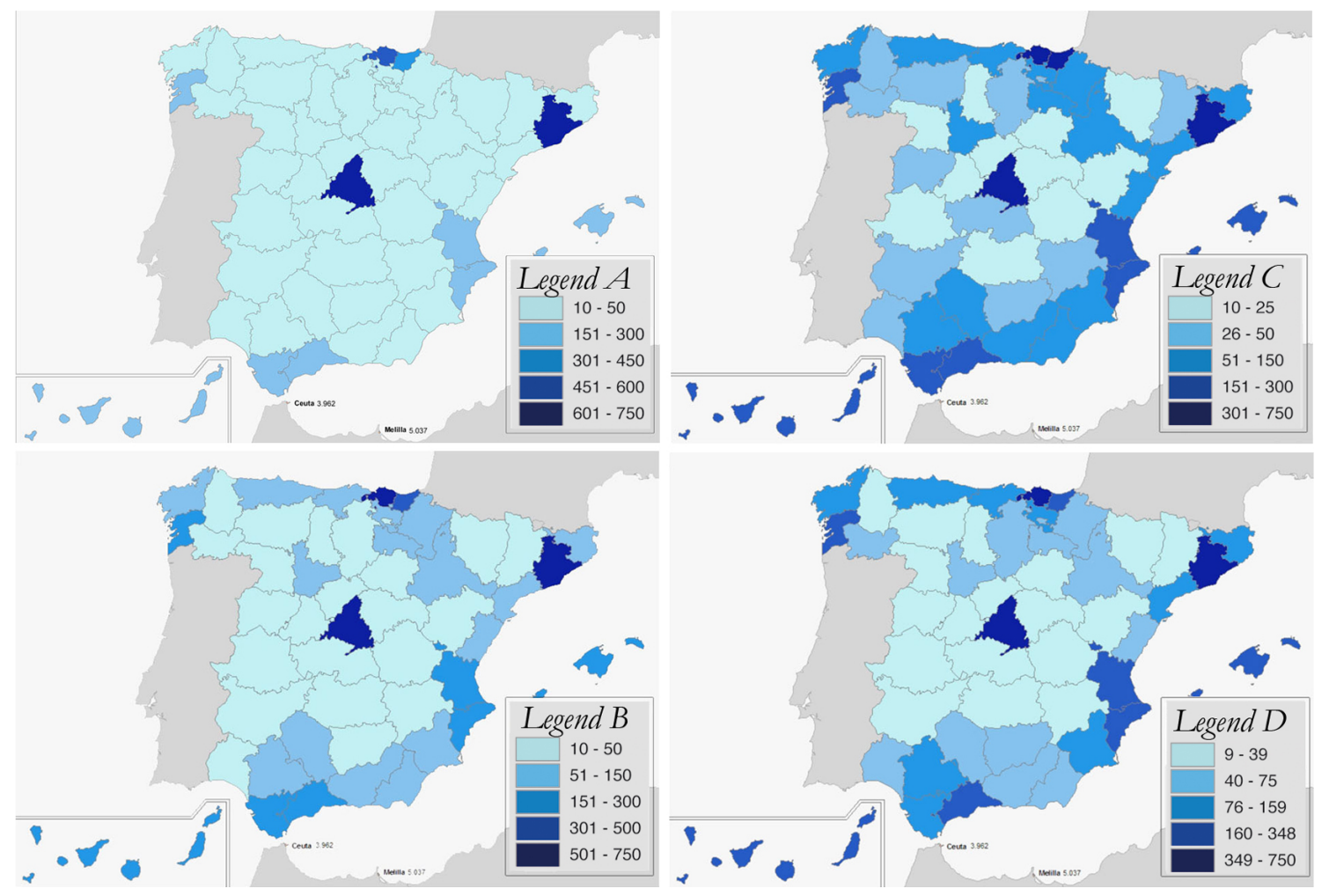

Fig. 7. Variability of class intervals depending on the number of classes and class limits. Different methods for class interval definition are shown: (A) equal class intervals: (B) arithmetic progression; (C) Geometric progression; and (D) Natural breaks intervals (Jenks).

research objectives. These are not related to the data and methods adopted, but to much more simple issues, such as how results are represented and displayed. All our rigorous scientific work might we misinterpreted if we do not know how to translate it to a proper language of the client or enduser. A field in which all these circumstances crop out is seismic risk assessment, because it involves professional users with a very different background and expectations as well as unprofessional users.

Different experience-based recommendations benefiting from well-established results of seismology and cartography are put together in this work with the objective of improving earthquake risk communication. Below, we summarize them and present extra conclusions derived from our work.

Regarding represented parameters:

- For professional users, present the results of the different parts of a seismic risk analysis are expressed in the same parameters that are contained in seismic codes or regulatory prescriptions, so that they can understand how they compare each other. Additionally for professional users, the combined use of relative and $a b$ solute parameters for representing the distributions of seismic vulnerability, expected damage and cost, is recommended. These parameters must be consistent with well-established classifications.
- For unprofessional users, results should be translated into simple classifications, such as high/medium/low vulnerability, light/intermediate/severe damage, etc. Specific values and parameters are turn out to be unnecessarily elaborated for this audience.

- Seismic hazard maps on rock conditions are only relevant for professional users. For unprofessional users it is recommended to provide a seismic hazard map including local effects.

- Cost estimates are relevant for all users: whereas cost/benefit ratios of accomplishment of pre- and postevent actions are suggested for professional users, relative estimates to average salary per capita or other more tangible parameter are recommended for unprofessional users.

Regarding graphical representations on maps:

- Maps should respect the natural perception that dark colours and bigger symbols represent higher values. Colourful figures do not imply a better outreach and should not be favoured. Map design should take care primarily of grey-value grading instead of hue variation. Accordingly, the traffic light criterion for representing seismic risk is not recommended, unless a careful consideration of the grey-value is taken. 
- A limited amount of class intervals (between three and five) are recommended for map design. It is important to thoroughly evaluate different choices for establishing the class intervals, as they control the final aspect of the map. Usually, natural breaks (Jenks intervals) are a good option, but this must be contrasted with the data.

- For professional users legends must contain all information of the represented parameters, whereas for unprofessional users qualitative legends are preferred because they are sufficiently informative and easy to understand.

Acknowledgements. The authors thank M. J. García Rodríguez, A. Rivas Medina, Y. Torres and B. Benito (Universidad Politécnica de Madrid) for the fruitful discussions. Comments to the final draft by M. ter Voorde (Vrije Universiteit Amsterdam) are highly appreciated. C. Lindholm and an anonymous referee are thanked for their reviews of the manuscript.

Edited by: L. Ferraris

Reviewed by: C. Lindholm and another anonymous referee

\section{References}

Anagnostopoulosa, S., Providakisb, C., Salvaneschic, P., Athanasopoulosa, G., and Bonacinad, G. SEISMOCARE: An efficient GIS tool for scenario-type investigations of seismic risk of existing cities, Soil Dyn. Earthquake Eng. 28, 73-84, 2008.

Bertin, J.: Sémiologie graphique, Les diagrammes, les réseaux et les cartes, Mouton, Gauthier-Villars, Paris, 431 pp., 1967.

Bertin, J.: Perception visuelle et transcription cartographique, International Yearbook of Cartography XVI, 25-43, 1976.

Bostenaru Dan, M.: Multidisciplinary co-operation in building design according to urbanistic zoning and seismic microzonation, Nat. Hazards Earth Syst. Sci., 5, 397-411, doi:10.5194/nhess-5397-2005, 2005.

Brewer, C. A.: Spectral schemes: controversial color use on maps, CaGIS 24, 203-220, 1997.

Cartwright, W.: New media and their application to the production of map products, Comput. Geosci., 23, 447-456, 1997.
Cauvin, C., Escobar, F., and Serradj, A.: Cartography and the Impact of the Quantitative Revolution, London, Ed. Wiley-ISTE, 432 pp., 2010.

Coburn, A. and Spence, R.: Earthquake Protection, John Wiley \& Sons, 2nd ed., 420 pp., 2002.

Dent, B. D.: Visual organisation and thematic map communication, Annals Ass. Am. Geographers, 62, 79-93, 1972.

Dent, B. D.: Cartography, Thematic Map Design, London: William C. Brown Publishers, Dubuque (USA), 5th edition, 448 pp., 1999.

Evans, I. S.: The selection of class intervals, Transactions of Institute of British Geographers 2, 98-123, 1977.

Geller, R. J., Jackson, D. D., Kagan, Y. Y., and Mulargia, F.: Earthquakes Cannot Be Predicted, Science, 275, 1616, 1997.

Gerstenberger, M. C., Wiemer, S., Jones, L. M., and Reasenberg, P. A.: Real-time forecasts of tomorrow's earthquakes in California, Nature, 434, 328-332, 2005.

Haque, C. E. and Etkin, D.: People and community as constituent parts of hazards: the significance of societal dimensions in hazards analysis, Nat. Hazards, 41, 271-282, 2007.

Jenks, G. F. and Coulson, M. R. C.: Class intervals for statistical maps, Intern. Yearbook of Cartography 3, 119-133, 1963.

MacEachren, A. M.: How maps work. Representation, visualization, and design, The Guilford Press, New York, 513 pp., 1995.

Mileti, D., Nathe, S., Gori, P, Greene, M., and Lemersal, L.: Public Hazards Communication and Education: The State of the Art. Update of Informer Issue 2: Public Education for Earthquake Hazards (originally published in 1999), 13 pp., 2004.

Miller, S.: Design of Multimedia Mapping Products, in: Multimedia Cartography, Springer Verlag Heidelberg, 89-104, 2007.

Petersen, M. P.: Elements of multimedia cartography,in: Multimedia Cartography. Springer Verlag Heidelberg, 63-73, 2007.

Reckelhoff-Dangel, C. and Petersen, D.: Risk communication in action: the risk communication workbook, US Environmental Protection Agency, 67 pp., 2007.

Robinson, A. H. and Petchenik B. B.: The map as a communication system, Cartographica, Monograph 19, 92-110, 1977.

Robinson, A. H., Morrison, J., Muehrcke, P. C., Kimerling, A. J., and Gutpill, S. C.: Elements of cartography, John Wiley \& Sons, New York, 4th ed., 448 pp., 1995.

Slocum, T. A., McMaster, R. B., Kessler, F. C., and Howard, H. H.: Thematic cartography and geography visualization, Pearson Prentice Hall, 518 pp., 2005. 